You might find this additional information useful...

This article cites 29 articles, 23 of which you can access free at:

http://ajpheart.physiology.org/cgi/content/full/280/2/H610\#BIBL

This article has been cited by 48 other HighWire hosted articles, the first 5 are:

Strain-Encoded CMR for the Detection of Inducible Ischemia During Intermediate Stress G. Korosoglou, S. Lehrke, A. Wochele, B. Hoerig, D. Lossnitzer, H. Steen, E. Giannitsis, N. F. Osman and H. A. Katus

J. Am. Coll. Cardiol. Img., April 1, 2010; 3 (4): 361-371.

[Abstract] [Full Text] [PDF]

Gap junction heterogeneity as mechanism for electrophysiologically distinct properties across the ventricular wall

M. Strom, X. Wan, S. Poelzing, E. Ficker and D. S. Rosenbaum

Am J Physiol Heart Circ Physiol, March 1, 2010; 298 (3): H787-H794.

[Abstract] [Full Text] [PDF]

Magnetic Resonance Tissue Phase Mapping of Myocardial Motion: New Insight in Age and Gender

D. Foll, B. Jung, E. Schilli, F. Staehle, A. Geibel, J. Hennig, C. Bode and M. Markl

Circ Cardiovasc Imaging, January 1, 2010; 3 (1): 54-64.

[Abstract] [Full Text] [PDF]

Role of Left Ventricular Twist Mechanics in the Assessment of Cardiac Dyssynchrony in Heart Failure

M. Bertini, P. P. Sengupta, G. Nucifora, V. Delgado, A. C.T. Ng, N. A. Marsan, M. Shanks, R. R.J. van Bommel, M. J. Schalij, J. Narula and J. J. Bax

J. Am. Coll. Cardiol. Img., December 1, 2009; 2 (12): 1425-1435.

[Abstract] [Full Text] [PDF]

\author{
Validation of 3-Dimensional Speckle Tracking Imaging to Quantify Regional Myocardial \\ Deformation \\ Y. Seo, T. Ishizu, Y. Enomoto, H. Sugimori, M. Yamamoto, T. Machino, R. Kawamura and K. \\ Aonuma \\ Circ Cardiovasc Imaging, November 1, 2009; 2 (6): 451-459. \\ [Abstract] [Full Text] [PDF]
}

Medline items on this article's topics can be found at http://highwire.stanford.edu/lists/artbytopic.dtl on the following topics:

Physics .. Magnetic Resonance

Physiology .. Left Ventricle

Medicine .. Etiology

Engineering .. Strain Analysis

Physiology .. Humans

Updated information and services including high-resolution figures, can be found at:

http://ajpheart.physiology.org/cgi/content/full/280/2/H610

Additional material and information about AJP - Heart and Circulatory Physiology can be found at: http://www.the-aps.org/publications/ajpheart

This information is current as of April 29, 2010 .

AJP - Heart and Circulatory Physiology publishes original investigations on the physiology of the heart, blood vessels, and lymphatics, including experimental and theoretical studies of cardiovascular function at all levels of organization ranging from the intact animal to the cellular, subcellular, and molecular levels. It is published 12 times a year (monthly) by the American

Physiological Society, 9650 Rockville Pike, Bethesda MD 20814-3991. Copyright @ 2005 by the American Physiological Society. ISSN: 0363-6135, ESSN: 1522-1539. Visit our website at http://www.the-aps.org/. 


\title{
Regional nonuniformity of normal adult human left ventricle
}

\author{
JAN BOGAERT ${ }^{1}$ AND FRANK E. RADEMAKERS ${ }^{2}$ \\ Departments of ${ }^{1}$ Radiology and ${ }^{2}$ Cardiology, University Hospitals, \\ Catholic University of Leuven, B-3000 Leuven, Belgium
}

Received 30 December 1999; accepted in final form 5 September 2000

\begin{abstract}
Bogaert, Jan, and Frank E. Rademakers. Regional nonuniformity of normal adult human left ventricle. Am J Physiol Heart Circ Physiol 280: H610-H620, 2001.-Regional nonuniformity is a feature of both diseased and normal left ventricles $(\mathrm{LV})$. With the use of magnetic resonance (MR) myocardial tagging, we performed three-dimensional strain analysis on 87 healthy adults in local cardiac and fiber coordinate systems (radial, circumferential, longitudinal, and fiber strains) to characterize normal nonuniformities and to test the validity of wall thickening as a parameter of regional function. Regional morphology included wall thickness and radii of curvature measurements. With respect to transmural nonuniformity, subendocardial strains exceeded subepicardial strains. Going from base to apex, wall thickness and circumferential radii of curvature decreased, whereas longitudinal radii of curvature increased. All of the strains increased from LV base to apex, resulting in a higher ejection fraction $(\mathrm{EF})$ at the apex than at the base $(70.9 \pm 0.4$ vs. $62.4 \pm 0.4 \%$; means $\pm \mathrm{SE}, P<0.0001)$. When we looked around the circumference of the ventricle, the anterior part of the LV was the flattest and thinnest and showed the largest wall thickening $(46.6 \pm 1.2 \%)$ but the lowest $\mathrm{EF}(64.7 \pm$ $0.5 \%)$. The posterior LV wall was thicker, more curved, and showed a lower wall thickening $(32.8 \pm 1.0 \%)$ but a higher $\mathrm{EF}(71.3 \pm 0.5 \%)$. The regional contribution of the $\mathrm{LV}$ wall to the ejection of blood is thus highly variable and is not fully characterized by wall thickening alone. Differences in regional LV architecture and probably local stress are possible explanations for this marked functional nonuniformity.
\end{abstract}

magnetic resonance; systolic function; morphology

EJECTION OF BLOOD IS THE RESULT of the inward motion of the myocardial wall, which is caused by the coordinated and sequential shortening of the sarcomeres within the myocytes of this wall. Sarcomere shortening can lead to ejection through different but related mechanisms at the macroscopic level: wall thickening, circumferential shortening, and longitudinal shortening. The relative contribution and regional nonuniformity of these different mechanisms in humans are largely unknown, because intrinsic myocardial deformation or strain could be studied only by using metallic markers, strain gauges, or ultrasonic devices surgically implanted in animals. However, with the introduction of noninvasive myocardial magnetic resonance (MR) tagging, studies in humans (both in healthy adults and

Address for reprint requests and other correspondence: J. Bogaert, Dept. of Radiology, Univ. Hospitals, Catholic Univ. of Leuven, Herestraat 49, B-3000 Leuven, Belgium (E-mail: Jan.Bogaert@uz. kuleuven.ac.be). patients) became feasible $(5,7,9,10,12,14,16,17,20$, $22,23,27-29)$. It is evident from these studies that the myocardial strain pattern is complex and closely related to the variations in transmural fiber orientation, irregular shape of the left ventricle $(\mathrm{LV})$, and local differences in ventricular morphology, i.e., radii of curvature and wall thickness.

Without the use of tags or implanted markers, wall thickening is the only strain that can be measured regionally, and as such it is often used as a parameter of regional contractile function. The purpose of this study was therefore to characterize in normal individuals regional strain in the local cardiac and fiber coordinate systems (radial, circumferential, longitudinal, and fiber strains and shears), as well as LV morphology and their interaction, and to define the relationship between wall thickening and regional ejection fraction (EF) as parameters of contractile behavior.

\section{METHODS}

\section{Study Population}

The study included 87 healthy adult humans (56 men and 31 women, $43.6 \pm 15.1 \mathrm{yr}$, range $22-74 \mathrm{yr}$ ) for MR tagging of the LV. Subjects were included if the clinical examination, echo Doppler examination, and stress test were normal. They were physically active (although not active athletes) and showed no evidence of obesity, chest wall abnormalities, coronary artery, valvular, or hypertensive disease. All of the participants were in normal sinus rhythm and none met electrocardiographic criteria for LV hypertrophy. None was taking medication known to influence cardiac function. All of the studies were performed according to the guidelines of the hospital ethics committee, and written informed consent was obtained from all of the volunteers.

\section{Study Protocol}

MR tagging. MR tagging was used to calculate myocardial strains (31). All of the MR tagging studies were performed with the use of a 1T MR unit (Magneton SP42, Siemens; Erlangen, Germany), by using an electrocardiographically gated and flow-compensated segmented $\mathrm{k}$ space FLASH gradient-recalled echo sequence with acquisition of $3 \mathrm{k}$ lines per heartbeat (repetition time: $14 \mathrm{~ms}$, echo time: $8 \mathrm{~ms}$, a variable flip angle, field of view: $400 \mathrm{~mm}$, matrix: $180 \times 256$, and slice thickness: $8 \mathrm{~mm}$ ). Eight time points were acquired for each studied cardiac level. The spread of these time points was

The costs of publication of this article were defrayed in part by the payment of page charges. The article must therefore be hereby marked "advertisement" in accordance with 18 U.S.C. Section 1734 solely to indicate this fact. 
nonequidistant but was adjusted to physiologically important phases during the cardiac cycle, e.g., end diastole, midsystole, end ejection, mitral valve opening, and fast filling. The exact timing of aortic valve closure was obtained with the use of a graphic auscultation system (Blood Line Technology; Incline Village, NV). Only measurements at end ejection are reported here.

Tags are noninvasive markers placed on the myocardium at end diastole by presaturating planes perpendicular to the subsequent imaging planes. The tags appear on the images as dark lines that move and deform with the myocardium on which they were inscribed (4) (Fig. 1). Five parallel short-axis planes and four radially oriented long-axis planes crossing in the center of the LV were defined (Fig. 2). This centerline of the LV cavity was obtained by the best fit connecting the centers of the cavity at each of the different short-axis levels, and its correct application was subsequently verified. The
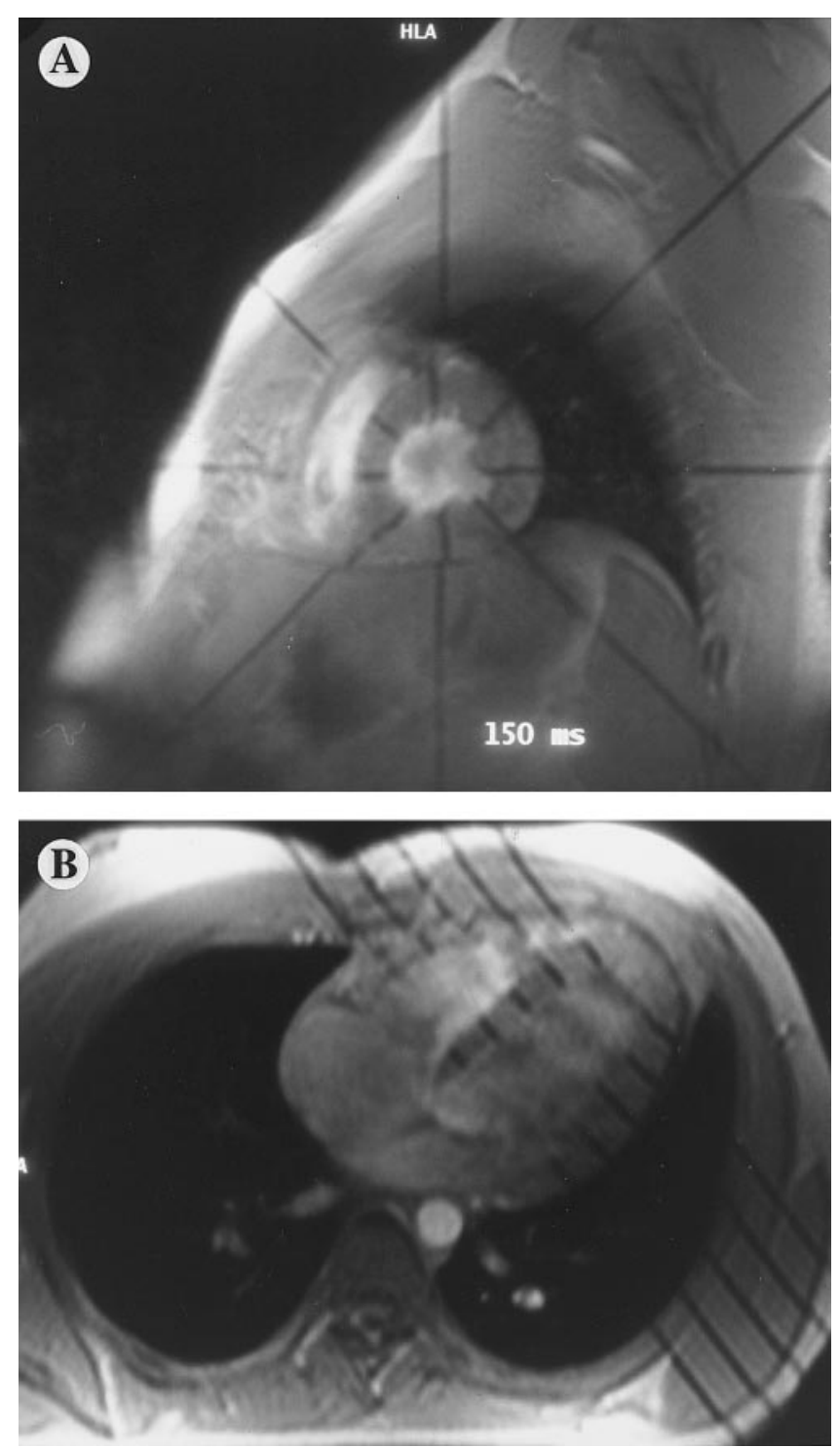

Fig. 1. Magnetic resonance (MR) tagging of the left ventricle (LV). $A$ : short-axis view at the midventricular level. $B$ : long-axis view or four-chamber view. In the short-axis plane, four tag lines are crossing each other in the center of the LV cavity. In the long-axis view, five tag lines are parallel and equally spaced from LV base to apex. images were acquired at end diastole and end ejection in all of these planes, by using the short-axis planes as tagging planes for the long-axis images and vice versa. By combining the short- and long-axis information, the entire LV wall, except for the apex, could be reconstructed into 32 small cuboids for which the three-dimensional coordinates of the node points were known.

Myocardial strains. Myocardial normal strain and shear are defined as the difference between an end-systolic and an end-diastolic dimension divided by a reference end-diastolic dimension and are, as such, dimensionless and presented as percent values. Strains are expressed as normal and shear strains (Fig. 3). Normal strains are defined as displacements along a coordinate axis. Two adjacent node points can be projected on a coordinate axis, and the length of this projection can be measured. The length of this projection can change during the cardiac cycle, and the difference of these lengths divided by the total length at end diastole defines the normal strains. Shear strains are similarly defined, but the difference in projected length and the end-diastolic distance by which it is divided are on different axes. In a local coordinate system, three normal and three shear strains are obtained. Normal strains will deform a cube into a beam, and shear strains will deform it into a parallelepiped.

Coordinate systems. These strains can be expressed in a local cardiac coordinate system and a local fiber coordinate system for each epicardial and endocardial node point (Fig. 2 ). Three perpendicular axes define the local cardiac coordinate system: radial $(R)$, circumferential $(C)$, and longitudinal $(L)$. The circumferential axis is obtained in a plane tangential to the inner or outer surface of the LV and oriented in the short-axis direction. The longitudinal axis is obtained in a similar plane but oriented along the LV long axis. The radial axis is obtained perpendicular to this tangential plane and directed outward by using the direction perpendicular to the wall (that is tilted near the apex because of the taper of the $\mathrm{LV})$. The local fiber coordinate system is defined by the following: 1$)$ the radial $(R)$ axis similar to the local cardiac coordinate system, 2) the fiber $(F)$ axis tangent to the surface and parallel to the local fiber orientation at either the epicardium or endocardium, and 3) the cross-fiber $(X)$ axis, tangent to the surface and perpendicular to the fiber. Fiber directions were obtained from histological fiber angle data in cadaver studies $(11,15,26)$. Myocardial strains can be negative or positive. Positive radial strains represent wall thickening, whereas negative strains represent segment shortening (e.g., circumferential shortening and fiber shortening).

Regional EF. Regional EF can also be quantified with MR myocardial tagging (Fig. 4). Because the short-axis tags are radially oriented with the crossing of the tag lines in the center of the LV cavity, each cuboid defines an intracavitary triangular volume, where the endocardial surface is the base of the triangle and the center of the LV is the apex. The inward motion and deformation (circumferentially and longitudinally) of the endocardium determine the changes in intracavitary triangular volume. Subtraction of the end-systolic from the end-diastolic triangular volume divided by the end-diastolic volume defines the regional EF (3). Similarly, the regional $\mathrm{EF}$ at the epicardium can be computed, but the changes in cuboid volume are added to the changes in intracavitary volume. Endocardial regional EF thus can be viewed as a composite measure of the local contribution to ejection. Because the composite measure is a percentage, it does not describe the absolute amount of ejected blood but rather the relative change at each cuboid. Near the apex, for example, the triangular volumes are somewhat smaller, and a larger 


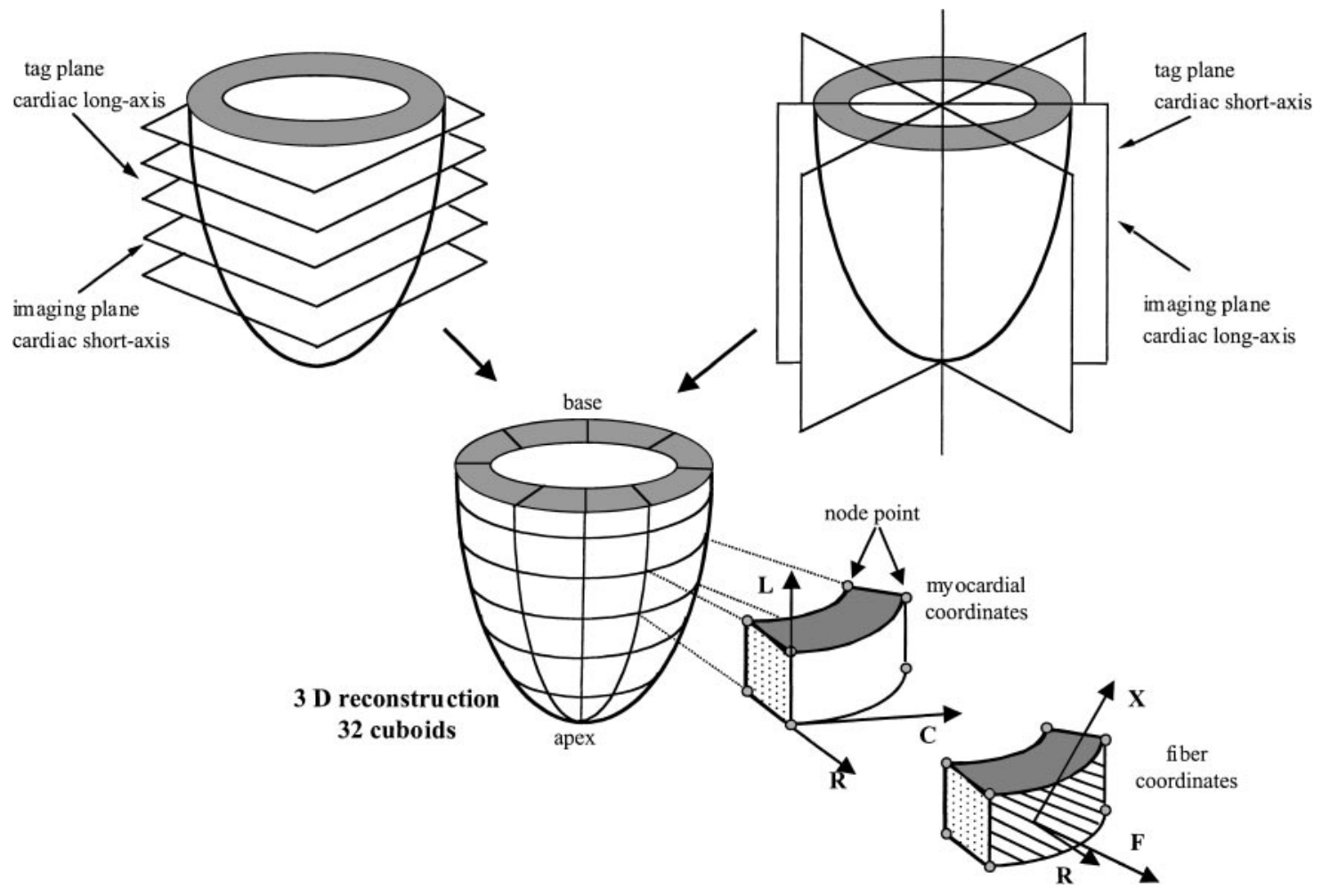

Fig. 2. Regional strain analysis with MR tagging. With a combination of MR tagging along the cardiac short and long axis, the LV wall is divided in 32 small cuboids. Each cuboid is defined by 4 epicardial and 4 endocardial node points. The strains are expressed in a local cardiac and local fiber coordinate system for each epicardial and endocardial node point. The axes used in the local cardiac coordinate system are radial $(R)$, by using the direction perpendicular to the wall; circumferential $(C)$, tangent to the surface and oriented in the short-axis direction for both the epicardium or endocardium; and longitudinal $(L)$, tangent to the surface and oriented in the cardiac long axis. The axes in the local fiber coordinate system are $R$, by using the direction perpendicular to the wall; fiber $(F)$, tangent to the surface and parallel to the local fiber direction at either the epicardium or endocardium; and cross-fiber $(X)$, tangent to the surface and perpendicular to $F$.

EF could still represent a smaller absolute volume change than at the base.

Myocardial wall thickness and circumferential and longitudinal radii of curvature. The true myocardial wall thick- ness is obtained in a three-dimensional fashion by adjusting the tag length for wall curvature in the longitudinal direction. The circumferential and longitudinal radii of curvature are calculated in each cuboid by using the chord and arch
Fig. 3. Normal and shear strains. "Nondeformed" myocardial cube is shown at end diastole (left). Subsequent myocardial deformation during systole (right), can be analyzed with a combination of three normal strains (middle) and three shear strains (right). EPI, epicardium; LR shear, longitudinal-radial shear strain; CL shear, circumferential-longitudinal shear strain; and $\mathrm{CR}$ shear, circumferential-radial shear strain.

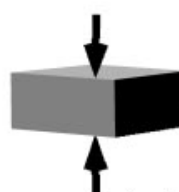

Longitudinal

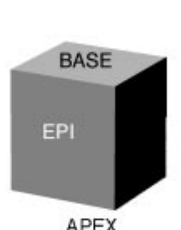

APEX

End Diastole
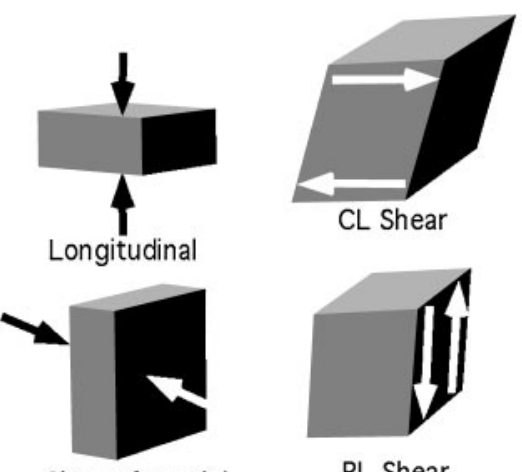

RL Shear
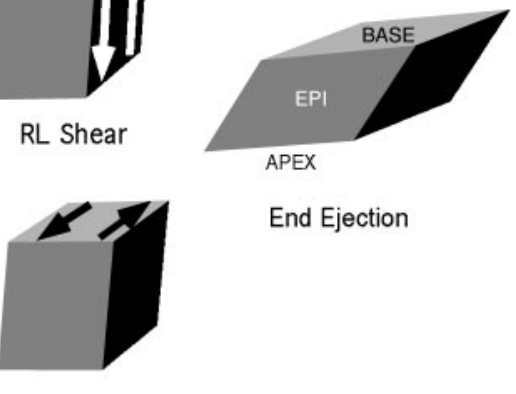

End Ejection

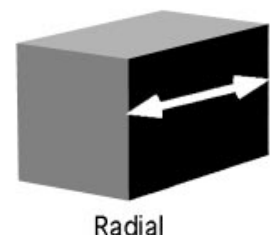

CR Shear 


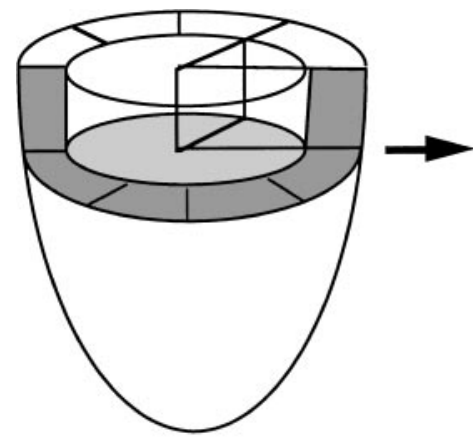

End diastole

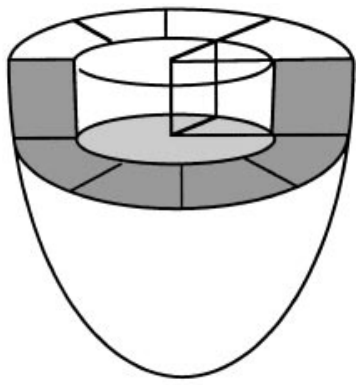

End ejection

Fig. 4. Regional ejection fraction (EF). The amount of blood ejected by each cuboid during systole is expressed by the regional $\mathrm{EF}$ at the endocardium. The regional EF at the epicardium can be computed by adding the changes in cuboid volume to the changes in intracavitary volume.

lengths fitting a model. A small radius of curvature denotes a curved surface, whereas a large radius of curvature is representative of a flat surface.

\section{Statistical Analysis}

For comparison of morphological and functional data, we used the unpaired Student's $t$-test and ANOVA with the Scheffe's post hoc test. In all of the cases, a value of $P<0.05$ was considered to indicate statistical significance. Unless stated otherwise, all data are means $\pm \mathrm{SE}$.

\section{RESULTS}

The regional results for morphology and strain are grouped as differences going through the wall, from base to apex and around the circumference of the ventricle.
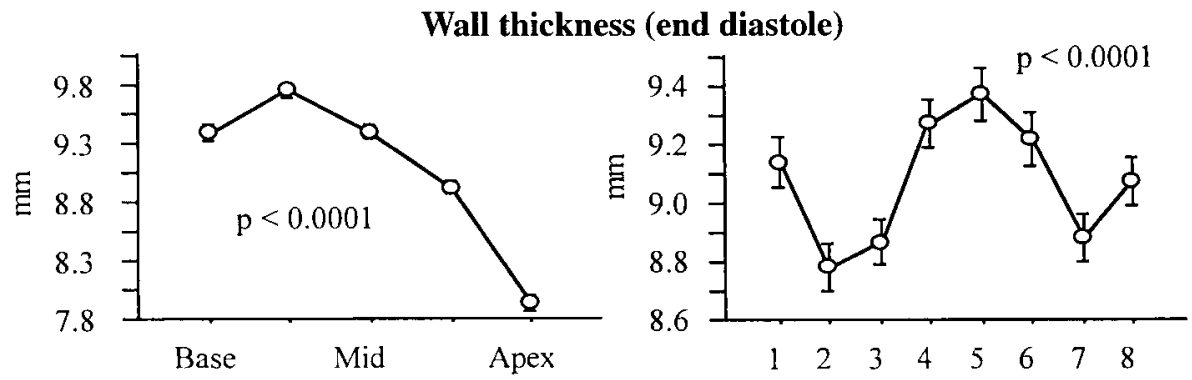

\section{Circumferential radius of Curvature (end diastole)}
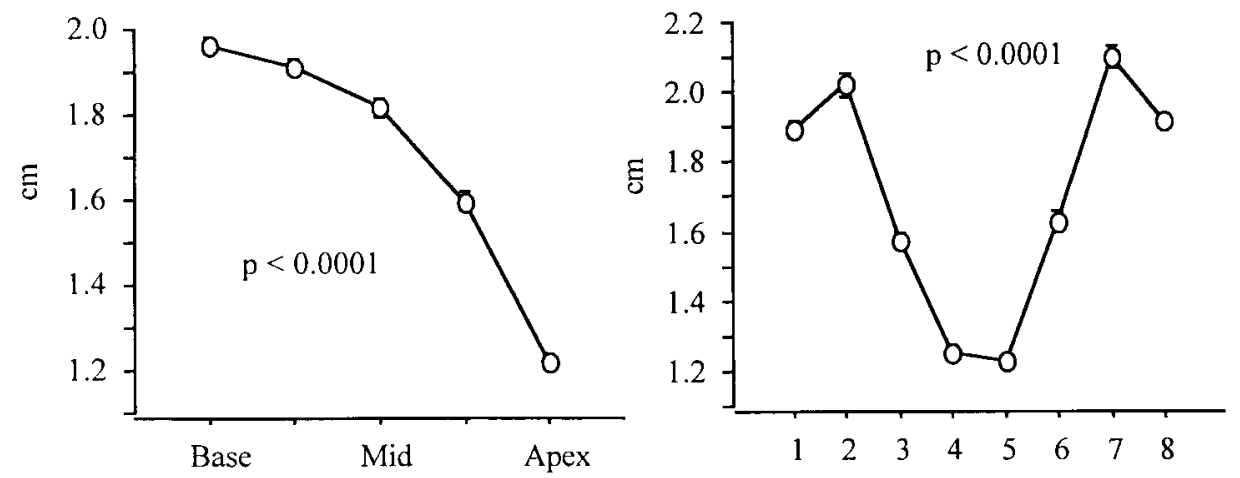

Fig. 5. Regional LV morphological parameters in base-to-apex direction (left) and around the LV circumference (right). In the circumferential direction, data were acquired at 8 locations and are shown counterclockwise, when viewed from apex to base: 1 , anteroseptal; 2, septal; 3, posteroseptal; 4, posterior; 5 , posterolateral; 6 , lateral; 7, anterolateral; and 8, anterior. In the longitudinal direction, 5 levels were acquired and are shown from base to apex. Results are expressed in millimeters and centimeters. $P<0.0001$, ANOVA.

\section{Longitudinal Radius of Curvature (end diastole)}
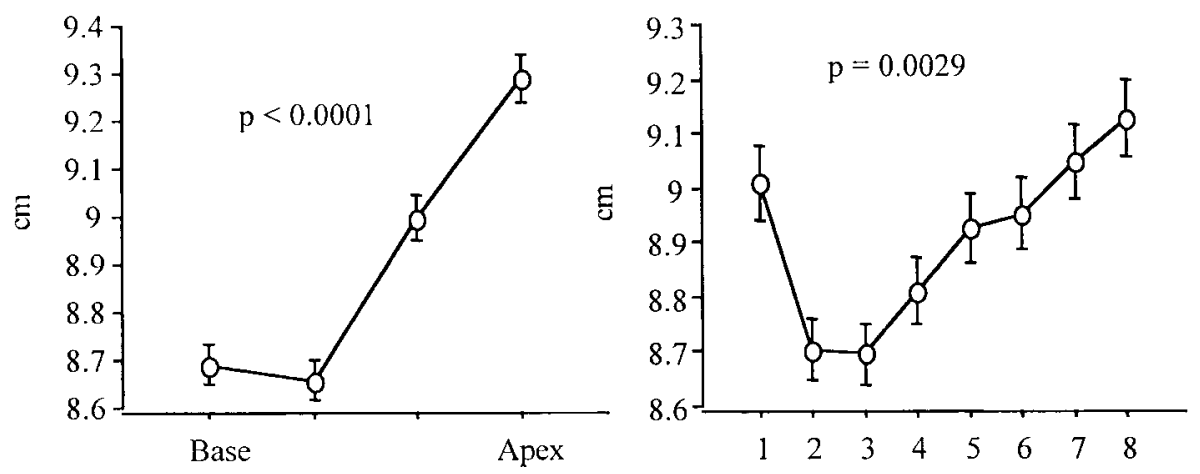


\section{Morphological Nonuniformities}

Transmural nonuniformity. The epicardial circumferential radius of curvature at end diastole was significantly larger than at the endocardium $(2.60 \pm 0.012$ epicardially vs. $1.70 \pm 0.010 \mathrm{~cm}$ endocardially, $P<$ 0.0001). There was no transmural difference in longitudinal radius of curvature at end diastole $(8.92 \pm$ 0.022 epicardially vs. $8.91 \pm 0.023 \mathrm{~cm}$ endocardially, $P=$ not significant, NS).

Base-to-apex nonuniformity. A significant difference in end-diastolic wall thickness was found $(P<0.0001)$ (Fig. 5). A gradual thinning of the LV wall was shown toward the apex with the exception of the most basal slice, which is also thinner $(P<0.001)$, probably because of the presence of the fibrous interventricular septum. Because of the conical shape of the LV, the end-diastolic circumferential radius of curvature gradually decreased from base to apex (from $1.96 \pm 0.02$ to $1.22 \pm 0.02 \mathrm{~cm}, P<0.0001)$. In contrast, the enddiastolic longitudinal radius of curvature increased toward the apex, expressing a flattening of the myocardium toward the apex (base: $8.69 \pm 0.04$, apex: $9.29 \pm$ $0.05 \mathrm{~cm}, P<0.0001)$.

Circumferential nonuniformity. The posterolateral wall was significantly thicker than the septum (9.37 \pm 0.07 vs. $8.78 \pm 0.05 \mathrm{~mm}, P<0.0001$ ) (Fig. 5). Moreover, at end diastole the LV circumference did not correspond to a circle but rather to an oval with a curved posterolateral wall and a flatter anterior wall and septum $(P<0.0001)$ (Fig. 5). Differences for the longitudinal radius of curvature were much less pronounced.

Table 1. Transmural differences in LV myocardial strain and regional ejection fraction

\begin{tabular}{lrrl}
\hline \hline & Epicardium & Endocardium & $P$ \\
\hline Circumferential strain & $-15.1 \pm 0.1$ & $-38.2 \pm 0.2$ & $<0.0001$ \\
Longitudinal strain & $-16.4 \pm 0.1$ & $-18.1 \pm 0.1$ & $=0.005$ \\
CL shear strain & $10.3 \pm 0.2$ & $9.2 \pm 0.2$ & $<0.0001$ \\
Fiber strain & $-17.9 \pm 0.2$ & $-23.7 \pm 0.2$ & $<0.0001$ \\
Cross-fiber strain & $-11.6 \pm 0.1$ & $-37.3 \pm 0.1$ & $<0.0001$ \\
Regional ejection fraction & $38.4 \pm 0.2$ & $66.9 \pm 0.2$ & $<0.0001$ \\
\hline
\end{tabular}

Values are means \pm SE. CL, circumferential-longitudinal; LV, left ventricular.

\section{Functional Nonuniformities}

Transmural nonuniformity. Although endocardial $\mathrm{EF}$ or inward motion is significantly larger than the corresponding epicardial measurement (endocardial EF: $66.9 \pm 0.2 \%$; epicardial EF: $38.4 \pm 0.2 \%, P<$ 0.0001) (Table 1), corresponding to the significant wall thickening, epicardial inward motion is substantial and significantly contributes to the ejection of blood. Related to the local cardiac coordinate system, transmural differences were most pronounced for circumferential shortening, much less so for longitudinal shortening with a slight excess in favor of endocardial shortening, thus leading to a positive longitudinalradial shear strain. Circumferential-longitudinal shear strains, expressing ventricular torsion, were significantly larger epicardially than endocardially $(P<$ 0.0001 ). According to the local fiber coordinate system, endocardial fiber shortening slightly exceeded epicardial fiber shortening $(17.9 \pm 0.2$ vs. $23.7 \pm 0.2 \%, P<$ $0.0001)$. The differences in cross-fiber strain were

\section{Base-to-Apex}

Epicardial

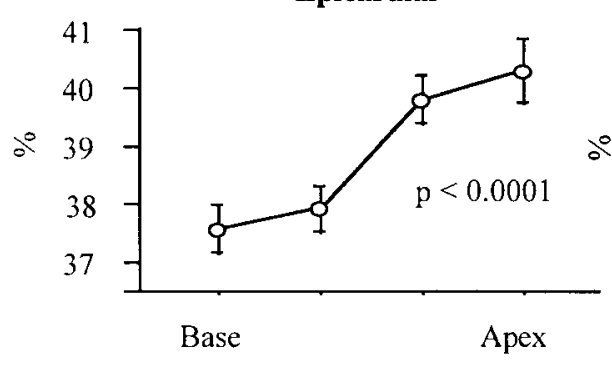

Endocardial

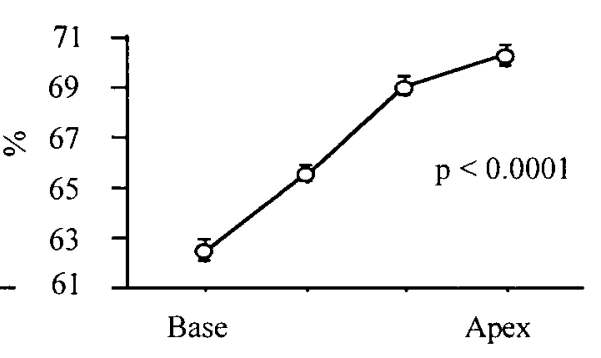

Circumference
Fig. 6. Regional EF in base-to-apex direction and around the LV circumference. In the circumferential direction, data are acquired at locations 1-8 (see Fig. 5). In the longitudinal direction, because the regional $\mathrm{EF}$ is a measurement calculated between adjacent levels, 4 values are obtained from base to apex. Results are expressed as percent values. $P<$ 0.0001 , ANOVA.

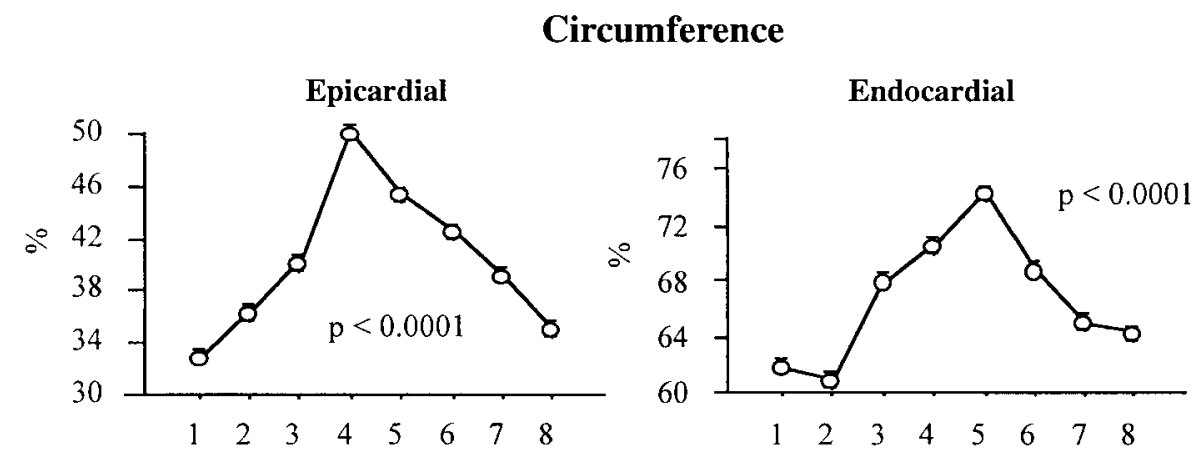


Table 2. Differences in myocardial strain in base-to-apex direction

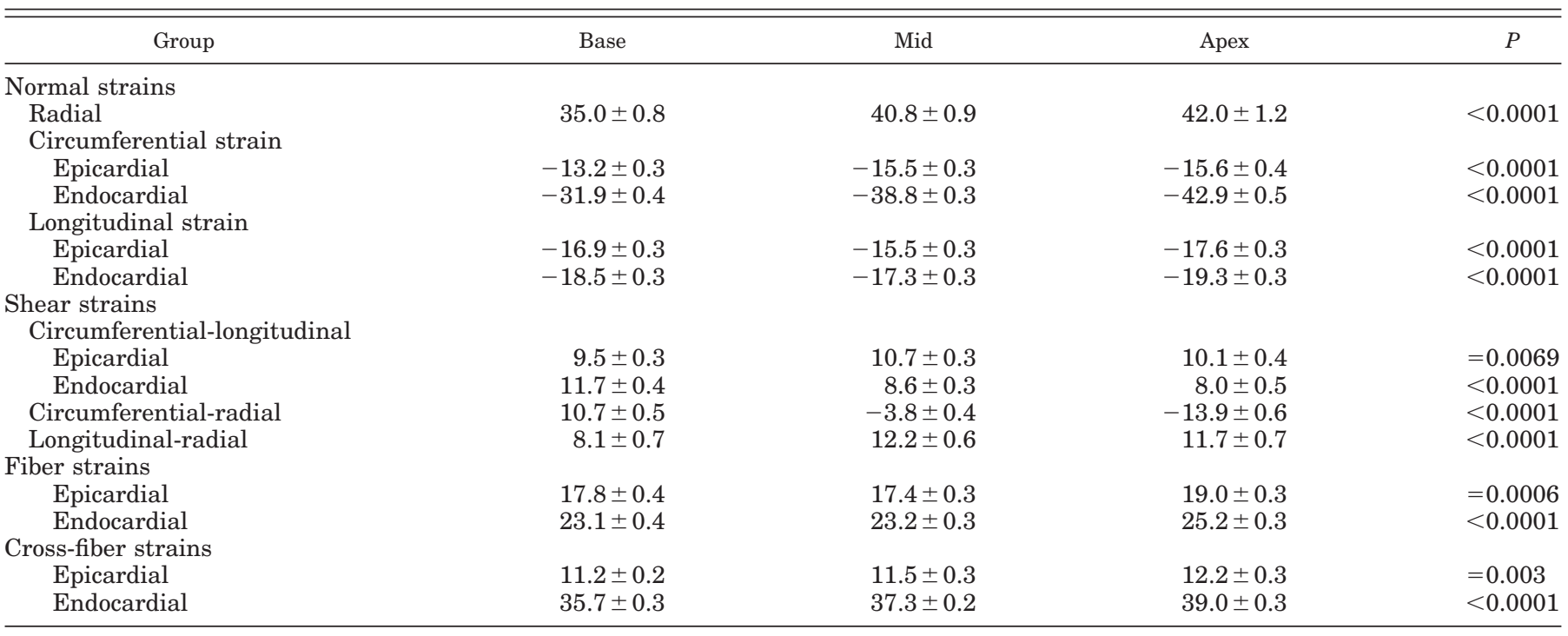

Values are means $\pm \mathrm{SE}$.

much more striking. At the epicardium, cross-fiber shortening was smaller than fiber shortening, whereas the endocardial cross-fiber shortening was almost twice as large as endocardial fiber shortening (epicardium: $11.6 \pm 0.1$ vs. endocardium: $37.3 \pm 0.1 \%, P<$ $0.0001)$.

Base-to-apex nonuniformity. The regional EF increased significantly from base to apex (Fig. 6 and Table 2). Increases in myocardial strain were most pronounced for wall thickening and endocardial circumferential shortening. A reversal in circumferentialradial shear strain was found. Fiber and cross-fiber shortening increased slightly but significantly from base to apex.

Circumferential nonuniformity. The posterior wall showed the largest endocardial EF but demonstrated the least systolic wall thickening (Fig. 6 and Table 3). This apparent contradiction could be explained by the larger epicardial circumferential shortening leading to a more pronounced centripetal global wall motion (Fig. 7). In contrast, the anterior wall and septum demonstrated the largest systolic wall thickening but had a smaller EF, which was primarily caused by the lower degree of circumferential and longitudinal shortening in this part of the LV wall. The endocardial circumferential-longitudinal shear strain, expressing ventricular torsion, was larger in the anterior wall compared with the posterior wall $(11.5 \pm 0.5$ and $6.3 \pm 0.6 \%$, respectively, $P<0.0001)$. In contrast, the longitudinalradial shear strain, expressing the relative motion of the inner versus the outer layers in longitudinal direction, was low in the anterior wall $(3.1 \pm 0.6 \%)$ but larger in the posterior wall $(17.2 \pm 0.9 \%)(P<0.0001)$. As shown in Fig. 8, significant differences in fiber and cross-fiber strain were found around the LV circumference. Remarkably, in the LV septum, epicardial fiber shortening (corresponds to RV endocardium) exceeded LV endocardial fiber shortening.

Table 3. LV myocardial strain: differences around the LV circumference

\begin{tabular}{|c|c|c|c|c|c|}
\hline & Anterior & Septum & Posterior & Lateral & $P$ \\
\hline \multicolumn{6}{|l|}{ Normal strains } \\
\hline Radial & $46.6 \pm 1.2$ & $38.9 \pm 1.0$ & $32.8 \pm 1.0$ & $35.8 \pm 1.1$ & $<0.0001$ \\
\hline \multicolumn{6}{|l|}{ Circumferential } \\
\hline Epicardial & $-12.7 \pm 0.3$ & $-12.2 \pm 0.3$ & $-22.1 \pm 0.4$ & $-16.1 \pm 0.3$ & $<0.0001$ \\
\hline Endocardial & $-36.2 \pm 0.5$ & $-34.0 \pm 0.4$ & $-41.6 \pm 0.5$ & $-39.3 \pm 0.5$ & $<0.0001$ \\
\hline \multicolumn{6}{|l|}{ Longitudinal } \\
\hline Epicardial & $-13.3 \pm 0.4$ & $-17.2 \pm 0.4$ & $-19.0 \pm 0.4$ & $-16.0 \pm 0.3$ & $<0.0001$ \\
\hline Endocardial & $-16.0 \pm 0.4$ & $-17.2 \pm 0.3$ & $-20.2 \pm 0.4$ & $-19.3 \pm 0.4$ & $<0.0001$ \\
\hline \multicolumn{6}{|l|}{ Shear strains } \\
\hline \multicolumn{6}{|c|}{ Circumferential-longitudinal } \\
\hline Epicardial & $9.9 \pm 0.4$ & $11.4 \pm 0.4$ & $10.2 \pm 0.5$ & $8.8 \pm 0.4$ & $<0.0001$ \\
\hline Endocardial & $11.5 \pm 0.5$ & $11.2 \pm 0.5$ & $6.3 \pm 0.6$ & $8.0 \pm 0.5$ & $<0.0001$ \\
\hline Circumferential-radial & $-4.0 \pm 0.8$ & $-2.9 \pm 0.6$ & $0.4 \pm 0.7$ & $0.4 \pm 0.8$ & $<0.0001$ \\
\hline Longitudinal-radial & $3.1 \pm 0.6$ & $12.6 \pm 0.7$ & $17.2 \pm 0.9$ & $9.3 \pm 0.7$ & $<0.0001$ \\
\hline
\end{tabular}

Values are means \pm SE. Data are shown for 4 of the 8 locations at the center of the following regions: anterior, septum, posterior, and lateral. 

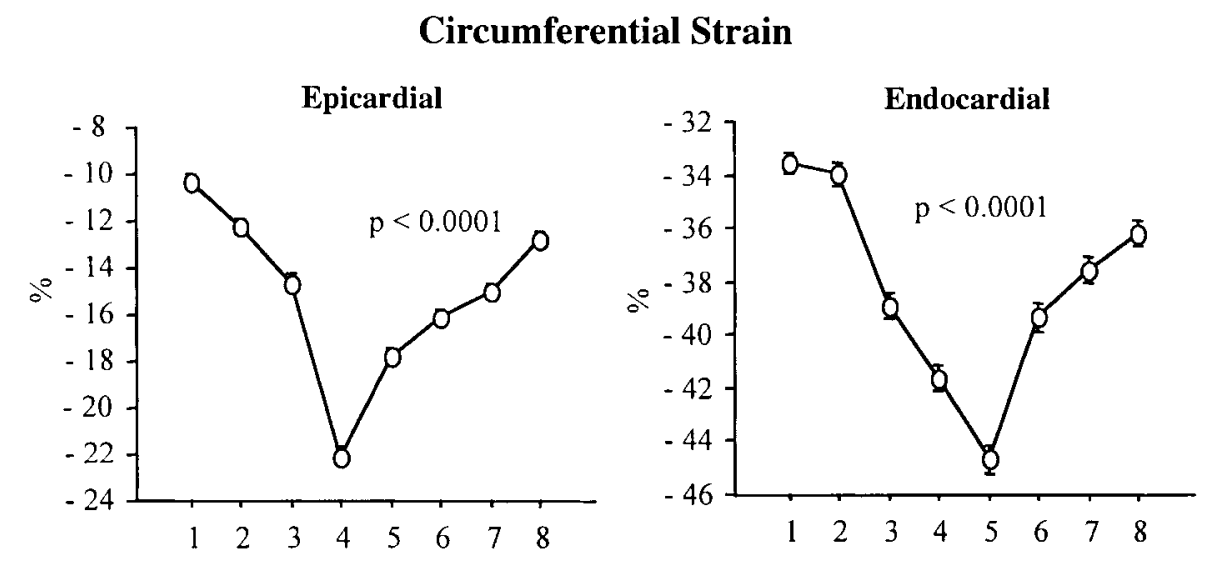

Fig. 7. Normal circumferential and longitudinal myocardial strains around the LV circumference. In the circumferential direction, data are acquired at locations 1-8 (see Fig. 5). Results are expressed as percent values. $P<$ 0.0001 , ANOVA.
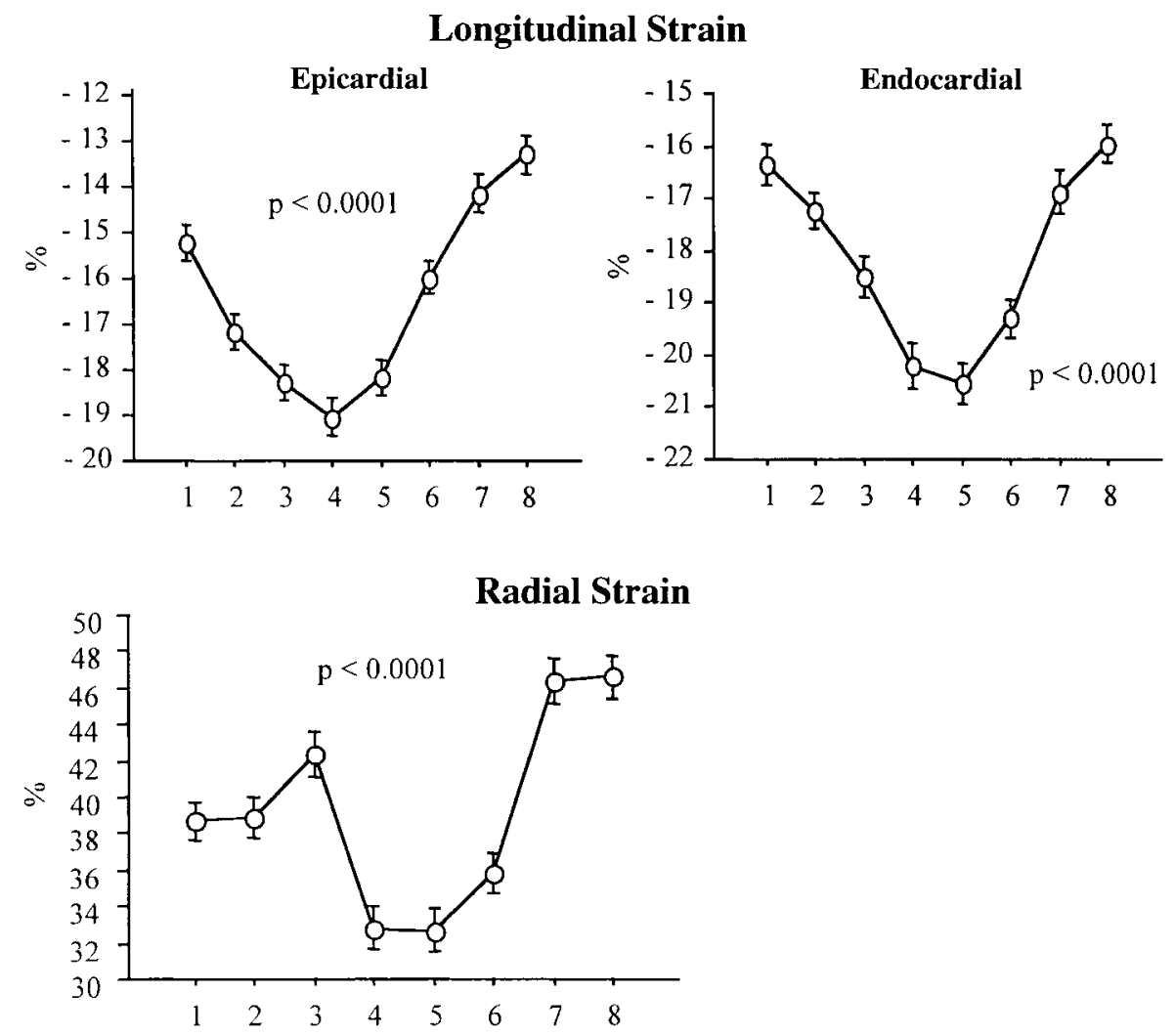

Relations Among Myocardial Wall Thickening, Regional EF, and Morphology

As shown in Fig. 9, a variable relation was shown between regional wall thickening and regional $\mathrm{EF}$, with $r$ values ranging from -0.58 to +0.64 . Thus regions with a higher wall thickening had a lower EF, except at the LV base. In contrast, an extremely tight positive relation was found between circumferential shortening and $\mathrm{EF}$ ( $r$ values ranging from 0.96 to 0.98 ), and also existed between longitudinal shortening and $\mathrm{EF}$ ( $r$ values ranging from 0.34 to 0.96 ). Regional EF correlated inversely with the regional endocardial radius of curvature at end diastole at most levels of the $\mathrm{LV}$ ( $r$ values ranging from -0.25 to -0.83 ). A highly variable relation was found between radii of curvature and myocardial wall thickening ( $r$ values in the range of -0.66 and 0.59 ).

\section{DISCUSSION}

With the use of MR myocardial tagging, regional morphology, strain, and function were quantified in 32 regions throughout the LV. The results demonstrate that the normal adult LV is characterized both morphologically and functionally by a high degree of regional nonuniformity. When endocardial regional EF is used as a reference measure of performance, circumferential shortening shows the closest relation, more so than longitudinal shortening, whereas thickening displays an opposite relation: less thickening is observed in the regions with a larger EF. Endocardial EF is also 


\section{Fiber Strain}
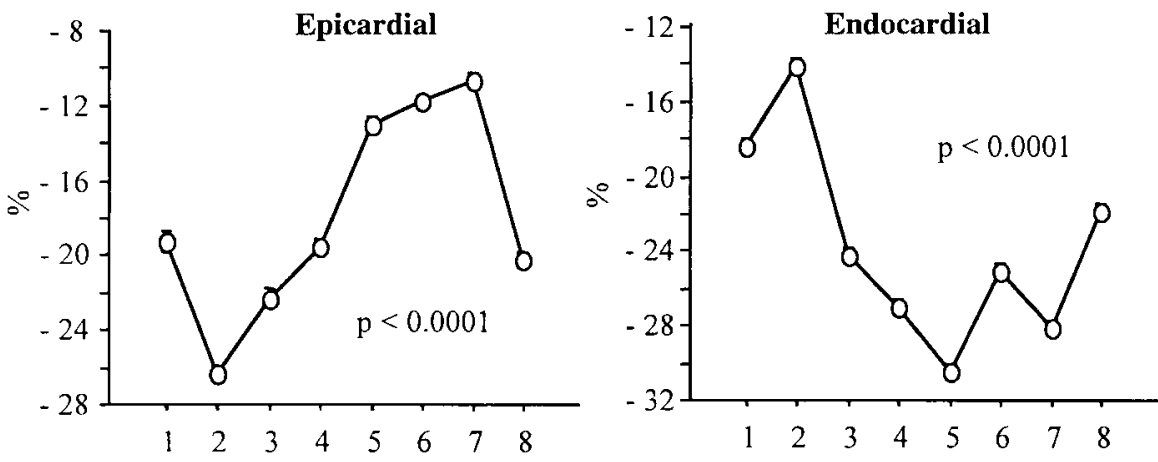

Fig. 8. Fiber strains and cross-fiber strains around the LV circumference. In the circumferential direction, data are acquired at loca-

Cross-Fiber-Strain
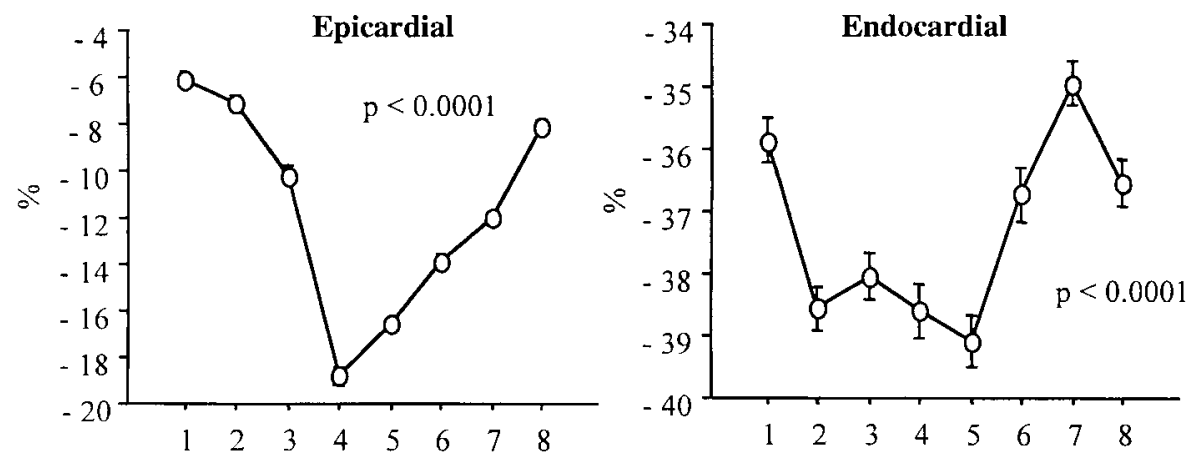

tions 1-8 (see Fig. 5). Results are expressed as percent values. $P<0.0001$, ANOVA.

better explained by regional morphology, i.e., radii of curvature, than is thickening.

\section{Morphological Nonuniformities}

First, the end-diastolic myocardial wall thickness varies significantly. The largest differences are found in the LV long-axis direction, whereas smaller differences are demonstrated around the LV circumference. These findings are in agreement with the anatomic data obtained by Greenbaum and colleagues (11). Differences in wall thickness are caused mainly by a variation in the amount of circumferentially oriented midwall fibers. Though abundant in the lateral walls, these fibers become very sparse toward the LV apex where the epicardial fibers lie in contact with the endocardial fibers. On the other hand, the thinning of the LV wall in its most basal regions may be caused by the inclusion of the membranous ventricular septum within the slice thickness. The differences in wall thickness around the LV circumference could be caused by a variation in the number of longitudinally oriented fibers. Second, whereas in most assumptions of wall stress the LV is compared with a thick-walled ellipsoid, the results of this study demonstrate that LV architecture is much more complex with large variations in thickness and circumferential and longitudinal radii of curvature. In the short-axis direction, the LV cavity has an oval rather than a circular configuration at end diastole. Moreover, in the long-axis direction, the LV wall flattens toward the apex. Thus more reliable estimates of regional LV wall stress have to take into account these variations in LV morphology, but on the other hand this variation, present in the normal ventricle, could be used to calculate regional stressstrain relations and so to quantify myocardial contractility without the need of preload or afterload changes.

\section{Functional Nonuniformities}

The deformation of the different myocardial segments not only varies transmurally but also from base to apex and around the circumference of the LV. Differences are most pronounced in the transmural direction, whereby most strains are significantly larger at the endocardium than at the epicardium, consistent with the principle of conservation of mass. Small variations exist in fiber strains, possibly explained by transmural differences in wall stress, not only at end diastole but also during systole. Estimates of LV wall stress have shown that the end-diastolic wall stress increases toward the endocardium (6). A larger fiber stretch leads to more fiber shortening during systole. This concept is supported by the observation that sarcomere lengths are shorter at epicardium than at endocardium (30). Though wall stress cannot be measured, Stein and colleagues (25) found a larger intramyocardial pressure in the endocardium than in the epicardium. Moreover, decreases in circumferential radii of curvature during systole are much more pronounced endocardially than epicardially. Thus subsequent wall stresses during ejection may be lower endocardially than epicardially and explain the higher endocardial fiber strains. A second explanation is the specific functional interaction among myocardial fiber layers (21). Compared with the canine LV, the human LV is more 
$\mathbf{A}$

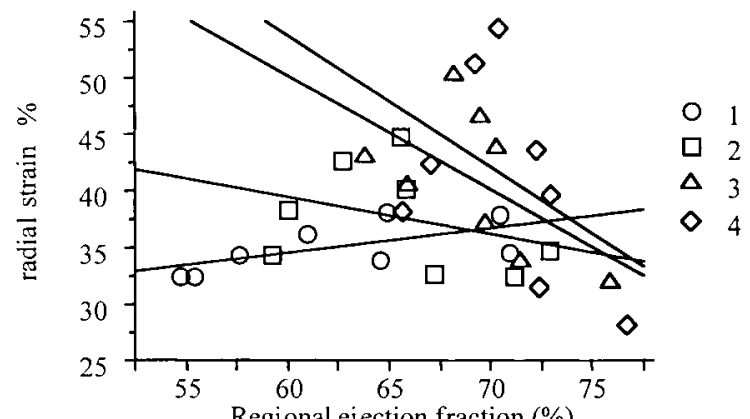

$\mathrm{Y}=.211+.002 * X, \mathrm{R}^{\wedge} 2=.407(1)$

$\mathrm{Y}=1.109-.01 * \mathrm{X}, \mathrm{R}^{\wedge} 2=.338(3)$

$\mathrm{Y}=.587-.003^{*} \mathrm{X}, \quad \mathrm{R}^{\wedge} 2=.113(2)$

B

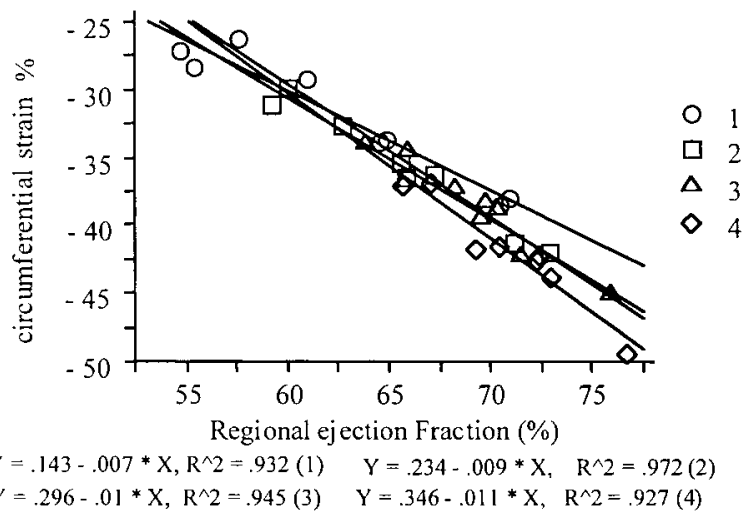

C

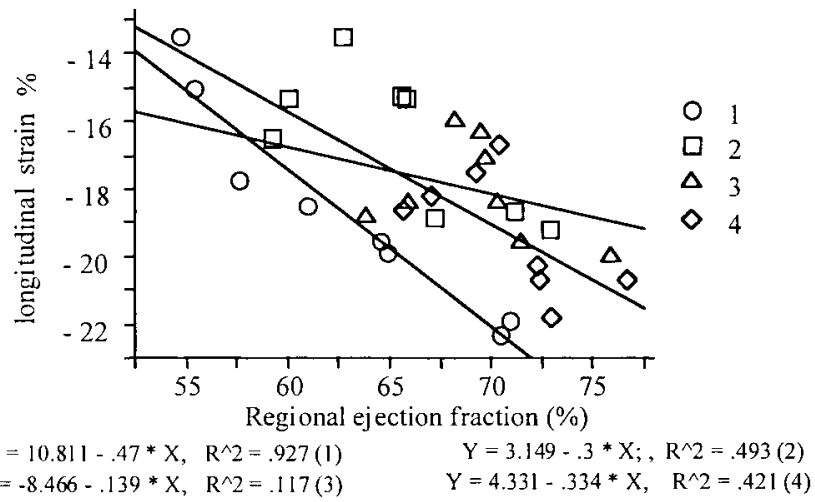

Fig. 9. Relation between regional EF and myocardial strains, i.e., radial strain or wall thickening $(A)$, circumferential strain $(B)$, and longitudinal strain $(C)$. Values are shown at 4 levels from base to apex: $\bigcirc$, most basal level; $\diamond$, most apical level.

conical and larger (torque difference between the epicardium and endocardium is smaller). This allows for less dominance of the epicardium over the endocardium and a more bidirectional interaction: more crossfiber shortening at the epicardium and less interference with endocardial fiber shortening. The latter could therefore explain the slightly larger endocardial fiber strains.

Another nonhomogeneity is found in the LV septum with a reversed pattern in fiber strain. Here, the strain in the outer layers exceeds the strain in the inner layers. The differences in strain in the septum are very likely related to the presence of the right ventricle. Because the outer myocardial layers in the septum represent the inner layers of the right ventricle working at much lower pressure values, this might be an important factor in the higher strain values.
The relative contribution to ejection increases toward the apex, although the myocardium significantly thins from LV base to apex. Because of the tapering of the LV walls, however, the absolute volume contribution of the LV apex is smaller. Again, differences in wall stress are the likely explanation for this discrepancy. On the basis of "simplified" wall-stress assumptions, Büchi and colleagues (6) showed a decrease in end-diastolic and mean systolic wall stress toward the apex. Because the ejection of blood is influenced by both preload and afterload and by myocardial contractility, one can understand that although the end-diastolic wall stress is lower in the apex (i.e., less fiber stretch), the regional function may be higher if the systolic wall stress is lower (for instance as a result of a smaller circumferential radius of curvature). Differences in myocardial morphology, such as the decrease of circumferentially oriented fibers, will very likely influence the degree of myocardial tethering. The increase in cross-fiber shortening toward the apex strengthens this hypothesis.

\section{Contribution of Myocardial Wall Thickening to Ejection of Blood}

Although several studies use myocardial wall thickening (i.e., radial strain) as the parameter to evaluate regional myocardial function in normal and pathological conditions, this study clearly illustrates the substantial contribution of circumferential inward motion and longitudinal shortening to the ejection of blood and the very variable contribution of wall thickening. This is most striking when evaluating the differences in regional EF around the LV circumference. Remarkably, the regions with the highest $\mathrm{EF}$ show the least wall thickening (i.e., posterior and lateral wall), whereas regions with lower EF show a greater wall thickening (i.e., anterior wall and septum). Conversely, a higher EF coincides with a larger epicardial inward motion (posterior epicardial circumferential shortening is twice that of the anteroseptal region). This relation between EF and thickening is not constant throughout the LV, however. This could be explained by two influencing factors, both related to LV architecture. Wall thickness and radius of curvature are important determinants of wall stress. A more curved wall will exhibit less wall stress, leading to a thinner wall at end diastole, which will show a relative larger thickening for the same amount of increase in wall thickness. On the other hand, a smaller systolic stress will allow a larger systolic regional EF. The balance between these two effects determines the final relation between thickening and EF. This is in contrast to the relation between EF and circumferential endocardial shortening, which is very tight in all of the regions, and this also holds to a lesser degree for longitudinal shortening. Different regions of the ventricle thus exhibit a variable contribution of thickening, global circumferential inward motion, and longitudinal shortening to the ejection of blood, and wall thickening certainly does not show the most simple, direct, and easy relation. Therefore, when wall thickening is used as a regional 
parameter of function, these complex relations have to be kept in mind when interpreting differences among patients and in one patient when comparing regions of the LV.

\section{Potential Limitations}

Several limitations of the present study need to be mentioned. A major objective of this study was a description of the regional morphology, strain, and function with MR tagging. The advantages and potential limitations of MR tagging have been described in other papers $(2,5,14,18,19,21)$. This technique is reliable for measuring strains of physiological magnitudes (1) and to study phenomena such as nonuniformities. However, the influence of other parameters such as nonhomogeneity in myocardial perfusion or myocardial innervation is unknown. Moreover, because in vivo determination of the fiber angle in living humans is impossible, the descriptions of the myocardial strain according to the epicardial or endocardial fiber direction were on the basis of fiber angles obtained in cadaver studies. Diffusion MR imaging has been reported to noninvasively determine the fiber angles in the in vivo heart $(8,13,24)$, but this technique is not available for routine use. Local or subtler variations of the fiber angles than those known from the cadaver studies may influence the data. Nevertheless, the results in the present study remain valid even when not related to a specific fiber direction.

An intrinsic shortcoming of the MR tagging technique, as used in this study, is the exclusion of the LV apex from the strain analysis. This is caused by the use of parallel orientation of tags in the long axis, whereby the most apical tag approaches the apex as closely as possible without covering the apex. Because the results in the present study demonstrate the increase in regional function toward the apex, further improvement of the radial tagging technique will be required to study this region more adequately. Finally, in the present study, we used end ejection as the time point to quantify the myocardial strain and function. This approach allowed us to express the nonuniform behavior during systole. However, further research will be required to evaluate whether nonuniformities in myocardial strain and function are present throughout the cardiac cycle (i.e., differences in activation of myocardial contraction) and how the interplay between regional and temporal nonhomogeneity occurs.

In conclusion, nonuniformity is a major characteristic of the normal adult human LV. Because the single myocyte behaves as a uniform structure, the results of the present study strongly suggest that the marked morphological nonuniformity has a decisive role in causing the heterogeneous myocardial deformation pattern. Wall thickening, often utilized as a marker of regional myocardial performance, has a very variable and difficult relation to other parameters of function and should be used with caution.

We thank H. Bosmans, G. Marchal, P. Suetens, L. Berben, and F. Van de Werf for support.
This study was supported by Nationaal Fonds voor Wetenschappelijk Onderzoek Grant G-3132.94.

\section{REFERENCES}

1. Azhari H, Weiss JL, Rogers WJ, Siu CO, Zerhouni EA, and Shapiro EP. Noninvasive quantification of principal strains in normal canine hearts using tagged MRI images in 3-D. Am $J$ Physiol Heart Circ Physiol 264: H33-H41, 1993.

2. Beyar R, Shapiro EP, Graves WL, Rogers WJ, Guier WH, Carey GA, Soulen RL, Zerhouni EA, Weisfeldt ML, and Weiss JL. Quantification and validation of left ventricular wall thickening by a three-dimensional volume element magnetic resonance imaging approach. Circulation 81: 297-307, 1990.

3. Bogaert J, Maes A, Van de Werf F, Bosmans H, Herregods M, Nuyts J, Desmet W, Mortelmans L, Marchal G, and Rademakers FE. Functional recovery of subepicardial myocardial tissue in transmural myocardial infarction after successful reperfusion. Circulation 99: 36-43, 1999.

4. Bosmans H, Bogaert J, Rademakers FE, Marchal G, Laub G, Verschakelen J, and Baert AL. Left ventricular radial tagging acquisition using gradient-recalled-echo techniques: sequence optimization. MAGMA 4: 123-133, 1996.

5. Buchalter MB, Rademakers FE, Weiss JL, Rogers WJ, Weisfeldt ML, and Shapiro EP. Rotational deformation of the canine left ventricle measured by magnetic resonance tagging: effects of catecholamines, ischaemia, and pacing. Cardiovasc Res 28: 629-635, 1994.

6. Büchi M, Hess OM, Murakami T, and Krayenbuehl HP. Left ventricular wall stress distribution in chronic pressure and volume overload: effect of normal and depressed contractility on regional stress-velocity relations. Basic Res Cardiol 85: 367-383, 1990.

7. Dong SJ, MacGregor JH, Crawley AP, McVeigh E, Belenkie I, Smith ER, Tyberg JV, and Beyar R. Left ventricular wall thickness and regional systolic function in patients with hypertrophic cardiomyopathy. Circulation 90: 1200-1209, 1994.

8. Edelman RR, Gaa J, Wedeen VJ, Loh E, Hare JM, Prasad $\mathbf{P}$, and $\mathbf{L i} \mathbf{W}$. In vivo measurement of water diffusion in the human heart. Magn Reson Med 32: 423-428, 1994.

9. Freeman GL, Le Winter MM, Engler RL, and Covell JW. Relationship between myocardial fiber direction and segment shortening in the midwall of the canine left ventricle. Circ Res 56: 31-39, 1985 .

10. Gallagher KP, Osakada G, Matsuzaki M, Miller M, Kemper WS, and Ross JJ. Nonuniformity of inner and outer systolic wall thickening in conscious dogs. Am J Physiol Heart Circ Physiol 249: H241-H248, 1985.

11. Greenbaum RA, Ho SY, Gibson DG, Becker AE, and Anderson RH. Left ventricular fibre architecture in man. $\mathrm{Br}$ Heart $J$ 45: 248-263, 1981.

12. Hansen DE, Daughters G, Alderman EL, Ingels NJ, and Miller DC. Torsional deformation of the left ventricular midwall in human hearts with intramyocardial markers: regional heterogeneity and sensitivity to the inotropic effects of abrupt rate changes. Circ Res 62: 941-952, 1988.

13. Hsu EW, Muzikant AL, Matulevicius SA, Penland RC, and Henriquez CS. Magnetic resonance myocardial fiber orientation mapping with direct histological correlation. Am J Physiol Heart Circ Physiol 274: H1627-H1634, 1998.

14. Lima JAC, Jeremy R, Guier W, Bouton S, Zerhouni EA, McVeigh E, Buchalter MB, Weisfeldt ML, Shapiro EP, and Weiss JL. Accurate systolic wall thickening by nuclear magnetic resonance imaging with tissue ragging: correlation with sonomicrometers in normal and ischemic myocardium. J Am Coll Cardiol 21: 1741-1751, 1993.

15. MacGowan GA, Shapiro EP, Azhari H, Siu CO, Hees PS, Hutchins GM, Weiss JL, and Rademakers FE. Shortening in the fiber and cross-fiber directions in the normal human left ventricle and in idiopathic dilated cardiomyopathy. Circulation 96: 535-541, 1997.

16. May Newman K, Omens JH, Pavelec RS, and McCulloch AD. Three-dimensional transmural mechanical interaction between the coronary vasculature and passive myocardium in the dog. Circ Res 74: 1166-1178, 1994. 
17. McCulloch AD, Smailland PJ, and Hunter BH. Left ventricular epicardial deformation in isolated arrested dog heart. $A m J$ Physiol Heart Circ Physiol 252: H233-H241, 1987.

18. McVeigh ER and Zerhouni EA. Noninvasive measurement of transmural gradients in myocardial strain with MR imaging. Radiology 180: 677-683, 1991.

19. Moore CC, Reeder SB, and McVeigh ER. Tagged MR imaging in a deforming phantom: photographic validation. Radiology 190: 765-769, 1994.

20. Prinzen FW, Arts T, van der Vusse GJ, Coumans WA, and Reneman RS. Gradients in fiber shortening and metabolism across ischemic left ventricular wall. Am J Physiol Heart Circ Physiol 250: H255-H264, 1986.

21. Rademakers FE, Rogers WJ, Guier WH, Hutchins GM, Siu CO, Weisfeldt ML, Weiss JL, and Shapiro EP. Relation of regional cross-fiber shortening to wall thickening in the intact heart. Three-dimensional strain analysis by NMR tagging. Circulation 89:1174-1182, 1994.

22. Rodriguez EK, Hunter WC, Royce MJ, Leppo MK, Douglas AS, and Weisman HF. A method to reconstruct myocardial sarcomere lengths and orientations at transmural sites in beating canine hearts. Am J Physiol Heart Circ Physiol 263: H293H306, 1992.

23. Rushmer RF, Crystal DK, and Wagner C. The functional anatomy of ventricular contraction. Circ Res 1: 162-170, 1953.

24. Scollan DF, Holmes A, Winslow R, and Forder J. Histological validation of myocardial microstructure obtained from dif- fusion tensor magnetic resonance imaging. Am J Physiol Heart Circ Physiol 275: H2308-H2318, 1998.

25. Stein P, Marzilli DM, Sabbach HN, and Tennyson L. Systolic and diastolic pressure gradients within the left ventricular wall. Am J Physiol Heart Circ Physiol 238: H625-H630, 1980.

26. Streeter DD. Gross morphology and fiber geometry of the heart. In: Handbook of Physiology. The Cardiovascular System. The Heart. Bethesda, MD: Am. Physiol. Soc., 1979, sect. 2, vol. I, chapt. 4, p. $61-112$.

27. Villarreal FJ and Lew WY. Finite strains in anterior and posterior wall of canine left ventricle. Am J Physiol Heart Circ Physiol 259: H1409-H1418, 1990.

28. Waldman LK, Fung YC, and Covell JW. Transmural myocardial deformation in the canine left ventricle. Normal in vivo three-dimensional finite strains. Circ Res 57: 152-163, 1985.

29. Waldman LK, Nosan D, Villarreal F, and Covell JW. Relation between transmural deformation and local myofiber direction in canine left ventricle. Circ Res 63: 550-562, 1988.

30. Yoran C, Covell JW, and Ross JJ. Structural basis for the ascending limb of left ventricular function. Circ Res 48: 297-303, 1973.

31. Zerhouni EA, Parish DM, Rogers WJ, Yang A, and Shapiro EP. Human heart: tagging with MR imaging-a new method for noninvasive assessment of myocardial motion. Radiology 169: 59-63, 1988.

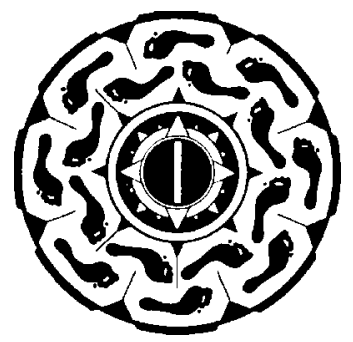

